\title{
PRODUÇÃO DE 'LEITE' DE SOJA ENRIQUECIDO COM CÁLCIO'
}

\author{
Fabiana CASÉ ${ }^{2}$, Rosires DELIZA ${ }^{3, *}$, Amauri ROSENTHAL ${ }^{3}$, Dilza MANTOVANI ${ }^{4}$, Ilana FELBERG ${ }^{3}$
}

\section{RESUMO}

"Leite" de soja é um produto de elevado valor nutricional, com alto conteúdo protéico, sendo um excelente produto para os indivíduos intolerantes à lactose. Entretanto, o conteúdo de cálcio - importante mineral para manutenção dos ossos - é baixo, sendo requerida sua adição, a fim de melhorar o valor nutricional do produto. O objetivo deste estudo foi produzir "leite" de soja adicionado de cálcio com adequada qualidade sensorial. A avaliação sensorial foi conduzida com uma equipe de provadores selecionados e treinados da EMBRAPA Agroindústria de Alimentos visando identificar o sal de cálcio mais adequado ao produto. Os seguintes atributos sensoriais foram analisados: gredosidade, sabor de feijão cru, corpo, sabor estranho e qualidade global de sabor. As perdas decorrentes do processamento também foram investigadas. O produto adicionado de fosfato tricálcio de sódio forneceu o melhor produto quanto às características sensoriais e o "leite" com lactato de cálcio apresentou as menores perdas durante o processamento, seguido do cálcio quelato. Entretanto, uma separação de fases foi observada no primeiro. Mesmo o "leite" de soja com cálcio quelato tendo apresentado sabor estranho, este foi o indicado para o enriquecimento, devido à alta solubilidade e biodisponibilidade.

Palavras-chave: 'leite' de soja; enriquecimento com cálcio; avaliação sensorial.

\section{SUMMARY}

PRODUCTION OF CALCIUM ENRICHED SOYMILK. Soymilk is a high nutritional product. It has high level of protein and it is an excellent substitute for those people who are not able to drink cow milk due to lactose intolerance. However, soymilk has low content of calcium, an important mineral for the production and maintenance of the bones. Consequently, it is required the addition of such mineral into the soymilk, in order to improve its nutritional value. This study aimed at producing soymilk added with calcium, with adequate sensory quality. Sensory tests were carried out by a selected and trained panel from Embrapa Food Technology to identify the more suitable calcium salt, which would be added to the product. The following sensory attributes were evaluated by the panel: chalkiness, beany flavour, body, off - flavour and global quality. The losses during the soymilk processing were investigated. Tricalcium phosphate yielded the highest sensory quality product for the trained panel. The sample with calcium lactate presented the lowest loss of the mineral during the processing, followed by the calcium quelate. However, it has been noticed a phase separation in the former. Despite the soymilk added with calcium quelate had presented off-flavour, it was chosen because this form of calcium is described as ideal to enrich food, due to its high solubility and bioavailability. The off-flavour perceived in this beverage by the assessors, can be masked using natural flavourings, such as strawberry, chocolate, and vanilla.

Keywords: soymilk; calcium fortification; sensory evaluation.

\section{1 - INTRODUÇÃO}

O interesse e a busca do consumidor por alimentos mais saudáveis propiciam um rápido crescimento do segmento da indústria de alimentos que visa contribuir para o alcance de uma dieta de melhor qualidade [9]. Os indivíduos estão mais preocupados com as conseqüências que o estilo de vida e os hábitos alimentares têm na sua saúde e bem-estar. A escolha e consumo inadequados de alimentos e dietas restritas podem afetar o estado nutricional do indivíduo em qualquer período de sua vida. Assim, oportunidades existem para que a indústria de alimentos manufature produtos que alcancem os requerimentos nutricionais de grupos populacionais específicos, tais como: crianças, adolescentes, mulheres, esportistas, idosos, etc. Atualmente, esse mercado de produtos ditos “naturais, saudáveis" é o segmento da indústria de alimentos que vem crescendo mais rapidamente, sempre associado aos pro-

\footnotetext{
Recebido para publicação em 22/05/2003. Aceito para publicação em 18/01/2005 (001136).

2. Departamento de Tecnologia de Alimentos, UFRRJ, Seropédica - RJ

3. EMBRAPA Agroindústria de Alimentos. Av. das Américas, 29.501, CEP 23.020-470; Rio de Janeiro-RJ. E.mail: rodeliza@ctaa.embrapa.br

4. ITAL - Av. Brasil, 2880 CEP 13073-001 Campinas - SP.

* A quem a correspondência deve ser enviada.
}

dutos que variam de modo de preparo rápido ao pronto para consumo (ready-to-eat), comparado àqueles que requerem maior tempo para sua preparação. Devido à imagem negativa do uso de remédios, e incertezas associadas à eficiência dos suplementos, a procura por alimentos funcionais tem se tornado bastante popular [21].

Segundo o Institute of Medicine's Food and Nutrition Board, entende-se por alimento funcional o produto alimentício que forneça beneficio específico à saúde, além dos tradicionais nutrientes nele contidos, isto é, alimentos que contém niveis significativos de componentes biologicamente ativos, os quais promovem beneficio ao indivíduo, além de simplesmente nutrir o organismo. Entre os alimentos funcionais mais estudados destacam-se a soja, o tomate, o peixe, a linhaça, as crucíferas (brócolis, couve de bruxelas, repo1ho), o alho e a cebola, as frutas cítricas, o chá verde, a uva/vinho tinto, a aveia, entre outros.

Alimentos funcionais não podem ser desenvolvidos simplesmente pela adição ou mistura de ingredientes apropriados. Efeitos decorrentes do processamento assim como os atributos sensoriais que afetam a qualidade final do produto devem ser considerados. A aparência, odor, textura e sabor têm importante papel na escolha e ingestão dos alimentos. Embora o corpo humano necessite de vários componentes para preservar sua inte- 
gridade estrutural e funcional, a grande maioria das pessoas come e bebe o que gosta, conferindo, assim, ao sabor, importante papel na escolha e consumo dos alimentos [11]. Entretanto, a ingestão de alimentos não é apenas dirigida por razões hedônicas, mas influenciada por uma série de fatores interrelacionados [22].

Soja e seus derivados têm sido utilizados há séculos nos países orientais como alimento básico da dieta daquelas populações, além de ingredientes para produtos industrializados no ocidente [7]. Pesquisas revelaram que a incidência e mortalidade causadas pelo câncer de mama em mulheres ocidentais tem sido consideravelmente mais elevadas que na Ásia, onde a soja tem importante papel na dieta [26]. Estudos epidemiológicos demonstraram que, além do câncer de mama e doenças cardiovasculares, a osteoporose, câncer de próstata e os sintomas da menopausa são raros nas sociedades asiáticas [17], demonstrando, assim, que a soja tem papel preventivo e terapêutico na saúde do indivíduo.

Para muitas pessoas o extrato hidrossolúvel de soja (EHS) - "leite" de soja - pode substituir o leite de vaca devido à intolerância ao leite bovino. Segundo o Instituto Nacional de Doenças Digestivas, Renais e Diabetes (EUA), cerca de $75 \%$ da população mundial é intolerante à lactose. A substituição do leite de vaca pelo EHS seria perfeita nutricionalmente, quando se referisse apenas à quantidade de proteína, porém ao considerarmos a quantidade dos micronutrientes, como por exemplo o cálcio, o "leite" de soja não se torna adequado substituto para o leite bovino, cujo conteúdo de cálcio é de $123 \mathrm{mg} / 100 \mathrm{~mL}$ de leite [12].

O consumo adequado de cálcio durante a vida é um pré-requisito para a saúde dos ossos. O cálcio é necessário para o desenvolvimento ósseo durante o crescimento e para a manutenção da integridade do esqueleto durante toda a vida adulta. Um dos mais importantes determinantes do risco de osteoporose pós-menopausa é a quantidade de massa esquelética adquirida durante a infância e adolescência. A deficiência de cálcio pode acarretar além da perda da massa óssea, problemas como cãibras e irritabilidade, por ser um mineral necessário na transmissão nervosa e na regulação dos batimentos cardiacos [10].

Biodisponibilidade de um mineral é a sua capacidade de estar biologicamente disponivel para ser absorvido pelo organismo humano. Diversos fatores afetam a biodisponibilidade do cálcio, tais como constituintes da dieta, fatores intestinais e tratamentos térmicos aplicados aos produtos já fortificados [4, 6, 18]. Um dos critérios para fortificação de produtos alimentícios é que o mineral usado resulte numa boa biodisponibilidade do elemento para o consumidor [23]. Quanto maior a solubilidade de um sal de cálcio maior a sua disponibilidade. A solubilidade de sais orgânicos é muito maior do que a dos sais inorgânicos. O lactato de cálcio é um sal orgânico solúvel e que não precipita com o calor [18]. A biodisponibilidade do cálcio nos vegetais pode ser afetada pelo seu conteúdo de fitatos e oxalatos, que são inibidores na absorção do cálcio. Em geral, a absorção de cálcio é inversamente proporcional ao conteúdo de ácido oxálico nos alimentos [27].

O enriquecimento do "leite" de soja com cálcio tem sido um tarefa dificil, pois os sais desse mineral podem promover coagulação das proteínas da leguminosa. O objetivo deste estudo foi desenvolver "leite" de soja enriquecido com sais de cálcio com adequadas características sensoriais.

\section{2 - MATERIAL E MÉTODOS}

As amostras de soja utilizadas neste trabalho foram da variedade IAS5, espécie Glycine max (L) Merril, pertencente à família das leguminosas, provenientes de Ponta Grossa (PR).

\section{1 - Enriquecimento e formulação do EHS com cál- cio}

A seleção dos sais de cálcio utilizados foi feita baseada em estudos de enriquecimento já existentes com o referido mineral [8, 14, 20, 22, 24, 25, 29], a saber: lactato de cálcio (Purac Sínteses), carbonato de cálcio (Merck), cálcio quelato (Albion), Foscalmix ${ }^{\circledR}$ e fosfato tricálcio (Coveg ${ }^{\circledR}$ ).

A quantidade de cálcio adicionada foi baseada na Legislação Brasileira, Portarias n 31 e 27 de 13 de janeiro de 1998, que determinam que vitaminas e minerais devem prover no mínimo $15 \%$ da Ingestão Diária Recomendada (IDR) em cada $100 \mathrm{~mL}$ dos produtos líquidos para serem classificados como alimentos enriquecidos de cálcio. Os diversos tipos de cálcio selecionados foram adicionados ao "leite" e estes foram submetidos à análise sensorial e análise de perdas durante o processamento, para identificar o produto mais adequado a ser utilizado na produção de "leite" de soja. As análises foram realizadas com nove amostras como mostra a Figura 1. A formulação das amostras seguiu o descrito na Tabela 1.

TABELA 1. Formulação das amostras de "leite" de soja enriquecido com cálcio.

\begin{tabular}{|c|c|c|c|c|}
\hline "Leite" de soja & $\begin{array}{c}\text { Sacarose } \\
(\%)\end{array}$ & $\begin{array}{l}\text { Cloreto de } \\
\text { sódio (\%) }\end{array}$ & $\begin{array}{l}\text { Citrato de } \\
\text { sódio (\%) }\end{array}$ & $\begin{array}{c}\text { Cálcio } \\
(\%)\end{array}$ \\
\hline Natural & 3 & 0,2 & - & - \\
\hline Adicionado de Cálcio quelato ${ }^{1}$ & 3 & 0,2 & - & 0,87 \\
\hline Adicionado de Lactato de cálcio ${ }^{2}$ & 3 & 0,2 & - & 0,85 \\
\hline $\begin{array}{l}\text { Adicionado de Lactato de cálcio }{ }^{2} \text { e } \\
\text { agente seqüestrante }^{3}\end{array}$ & 3 & 0,2 & 0,48 & 0,85 \\
\hline Adicionado de Carbonato de cálcio ${ }^{4}$ & 3 & 0,2 & - & 0,30 \\
\hline $\begin{array}{l}\text { Adicionado de Carbonato de cálcio }{ }^{4} \\
\text { e agente seqüestrante }\end{array}$ & 3 & 0,2 & 0,06 & 0,30 \\
\hline Adicionado de Foscalmix ${ }^{\circledast 5}$ & 3 & 0,2 & - & 0,30 \\
\hline Adicionado de Fosfato tricálcio ${ }^{6}$ & 3 & 0,2 & - & 0,30 \\
\hline $\begin{array}{l}\text { Adicionado de Fosfato tricálcio }{ }^{6} \text { e } \\
\text { agente seqüestrante }\end{array}$ & 3 & 0,2 & 0,06 & 0,30 \\
\hline
\end{tabular}

${ }^{1}$ Registrado como "Milkcal" pela Albion ${ }^{\circledast} ;{ }^{2}$ Purac $^{\circledast} ;{ }^{3}$ Reagen $^{\circledast}$ - Quimibrás Indústria Quimica S.A; ${ }^{4}$ Merck $^{(\mathbb{m} ;}{ }^{5}$ Associação do Fosfato Tricálcio com um espessante - Coveg - Fosmix Fosfatos e Misturas Alimentícias Indústria e comércio Ltda.; ${ }^{6}$ Coveg $^{\mathbb{1}}$ 


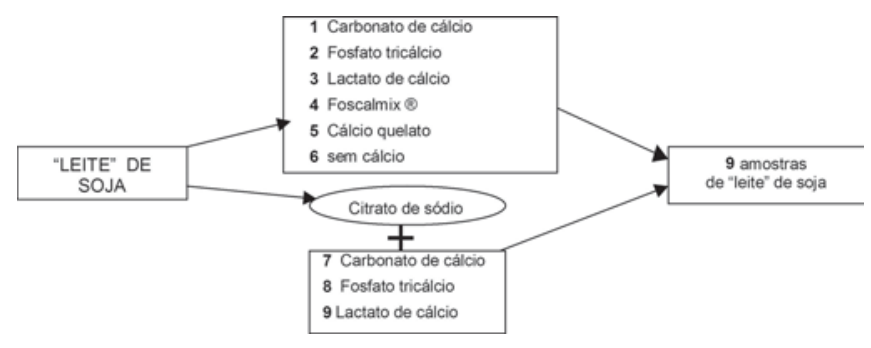

FIGURA 1. Obtenção das amostras de "leite" de soja.

\section{2 - Determinação de cálcio}

Amostras experimentais dos nove extratos hidrossolúveis de soja (Figura 1) foram retiradas durante três etapas do processamento (Figura 2) baseado no estudo de NELSON et al. [19], isto é: após formulação, após o aquecimento e no produto final, logo após homogeneização. Todas os tratamentos foram adicionados de 240mg cálcio/100mL de bebida (30\% da IDR, quantidade acima da recomendada para compensar as possiveis perdas durante o processamento). As amostras foram mantidas congeladas a $-18^{\circ} \mathrm{C}$, sendo posteriormente descongeladas e homogeneizadas, para a análise do mineral, a qual foi realizada em triplicada.

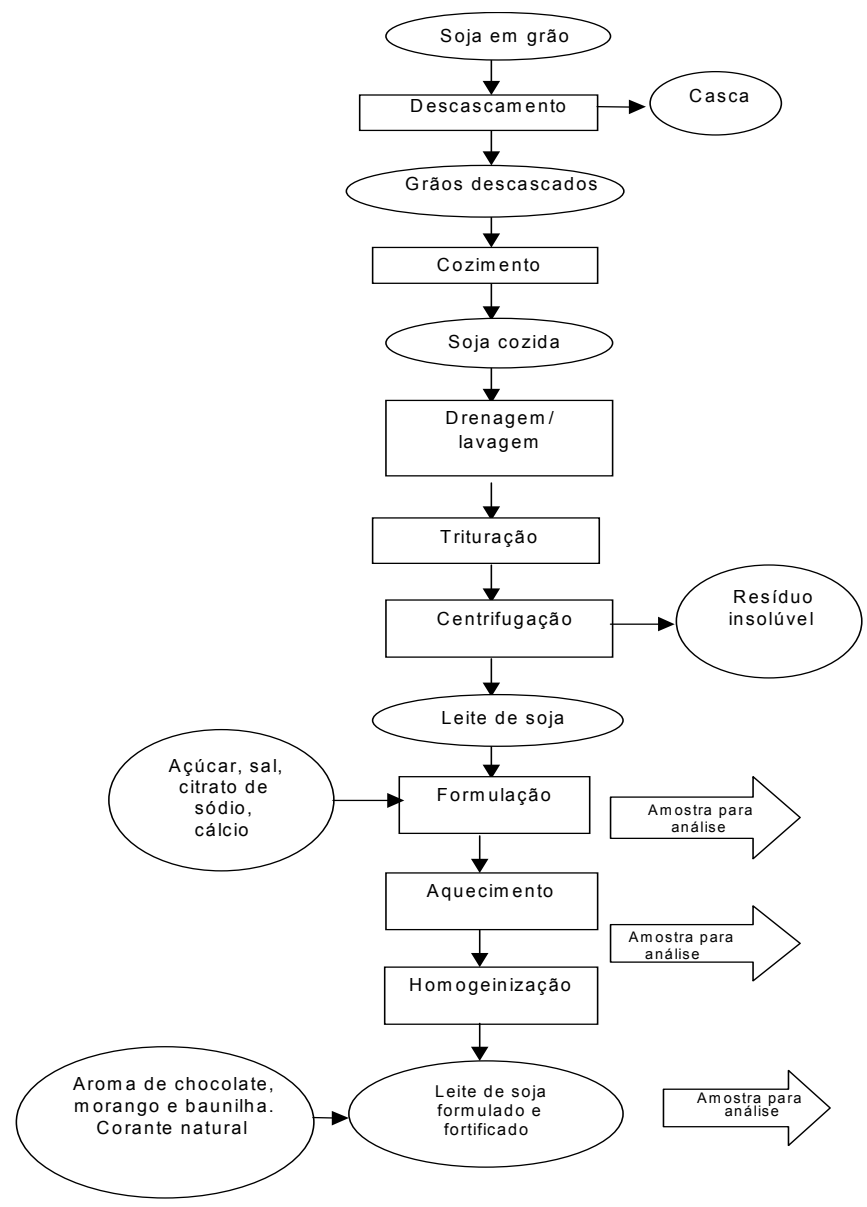

FIGURA 2. Fluxograma da produção do "leite" de soja enriquecido com cálcio segundo CASÉ et al. [5].
A determinação do cálcio foi realizada no Instituto de Tecnologia de Alimentos - ITAL, usando o método de mineralização por via seca segundo AOAC [6] e quantificação pela técnica de espectrometria de emissão atômica em plasma indutivamente acoplado.

\section{3 - Análise sensorial}

A análise sensorial foi realizada por uma equipe de provadores selecionados e treinados da EMBRAPA Agroindústria de Alimentos. Tal equipe foi constituída de nove provadores (quatro mulheres e cinco homens) com idades entre 25-42 anos que participavam com freqüência dos testes sensoriais no laboratório de Análise Sensorial. Após várias sessões de treinamento, foram levantados os atributos usados na análise, de acordo com a terminologia sugerida pela equipe. Foram definidos os extremos da escala utilizada e então elaborou-se a ficha usada neste estudo sensorial. Finalmente, os provadores foram treinados para quantificar a intensidade das sensações percebidas. Os provadores avaliaram as nove amostras do "leite" de soja, sendo uma delas denominada padrão (sem adição do mineral) e as demais oito amostras adicionadas de algum tipo de sal de cálcio.

As amostras foram avaliadas quanto à gredosidade, "beany flavour", corpo, sabor estranho e qualidade global utilizando escala não estruturada de 9 pontos, com a intensidade variando de fraco a forte, onde os provadores marcavam com um traço vertical a intensidade percebida em cada amostra. Foram oferecidos cerca de $30 \mathrm{~mL}$ de cada "leite" a temperatura ambiente, seguindo delineamento de blocos completos balanceados com duas repetições. As amostras foram apresentadas aos provadores monadicamente, três por sessão nas cabines individuais sob luz vermelha, acompanhado de água mineral para limpar o palato.

Os dados foram analisados estatisticamente através da Análise de Variância e teste de Tukey para checar a diferença entre médias, ao nível de $5 \%$ de probabilidade.

\section{4 - Composição centesimal}

As amostras foram submetidas à liofilização e enviadas ao Instituto de Tecnologia de Alimentos - ITAL para as seguintes análises: proteínas, de acordo com método do American Association of Cereal Chemists [1] - método 46-12; lipídios totais por hidrólise, seguindo as normas do método da AOAC [15]; umidade, seguindo metodologia do Instituto Adolfo Lutz [16]; cinzas: seguindo metodologia descrita na AOCS [3], recomendada para análise de leite; carboidratos: obtidos por diferença, onde 100 - (g/100g umidade + g/100g lipídios totais + g/ 100g proteína bruta); calorias: o valor calórico foi calculado pela soma das porcentagens de proteína bruta e carboidratos multiplicado pelo fator 4 (kcal/g) somado com o teor de lipídios totais multiplicado pelo fator 9 (kcal/g). 


\section{3 - RESULTADOS E DISCUSSÃO}

\section{1 - Avaliação da perda de cálcio durante o pro- cessamento}

A quantidade de cálcio em cada etapa do processamento variou, como mostra a Tabela 2 . Tal variação pode ser explicada devido à solubilidade de cada sal de cálcio, a qual pode variar de acordo com a temperatura e o $\mathrm{pH}[23,24,28]$. Além disso, não se pode excluir um provável erro operacional ocorrido na etapa na qual adicionou-se o cálcio seguido de aquecimento à $80^{\circ} \mathrm{C}$.

Analisando a Tabela 2, observa-se que as amostras adicionadas de fosfato tricálcio, com e sem citrato, apresentaram na etapa da formulação, quantidade de cálcio correspondente a $91,82 \mathrm{mg}$ e $77,84 \mathrm{mg}$ respectivamente, inferior, portanto, à quantidade adicionada de $240 \mathrm{mg}$. O teor de cálcio aumentou nas etapas subseqüentes chegando a 168,02mg na amostra adicionada de fosfato tricálcio com citrato e 163,12mg nas amostras sem citrato. Este resultado pode ser devido à solubilidade deste sal de cálcio. Segundo WEINGARTNER et al. [28], o fosfato tricálcio é insolúvel em água fria enquanto o citrato de cálcio é extremamente solúvel, ou seja, a temperatura da amostra na qual adiciona-se o sal de cálcio foi fator importante na solubilidade.

TABELA 2. Teor de cálcio (mg/100g) nas amostras de "leite" de soja (EHS) nas diferentes etapas do processamento.

\begin{tabular}{|c|c|c|c|}
\hline \multirow{3}{*}{ Amostras } & \multicolumn{3}{|c|}{ Etapas do Processamento $^{B}$} \\
\hline & \multirow{2}{*}{ FormulaÁ, o } & ApÛs & \multirow{2}{*}{$\begin{array}{c}\text { ApÛ́s } \\
\text { HomogeneizaÁ, o }\end{array}$} \\
\hline & & Aquecimento & \\
\hline EHS Natural $^{1}$ & 8,39 & 8,64 & 8,64 \\
\hline EHS + Carbonato & & 17404 & \\
\hline $\mathrm{Ca}^{++}$com Citrato & $1 / 8,44$ & $1 / 4,04$ & 156,58 \\
\hline $\begin{array}{l}\text { EHS + Fosfato tric } \cdot \text { Icio } \\
\text { com Citrato }\end{array}$ & 91,82 & 157,96 & 168,02 \\
\hline $\begin{array}{l}\mathrm{EHS}+\text { Lactato de } \mathrm{Ca}^{++} \\
\text {com Citrato }\end{array}$ & 191,94 & 376,62 & 234,88 \\
\hline EHS + c. Icio Quelato & 185,96 & 223,28 & 225,14 \\
\hline EHS + Foscalmixf & 170,52 & 201,52 & 172,44 \\
\hline $\mathrm{EHS}+$ Carbonato $\mathrm{Ca}^{++}$ & 144,48 & 111,12 & 126,78 \\
\hline EHS + Fosfato tric $\cdot$ Icio & 77,84 & 139,88 & 163,12 \\
\hline $\mathrm{EHS}+$ Lactato $\mathrm{Ca}^{++}$ & 185,80 & 230,40 & 235,80 \\
\hline
\end{tabular}

${ }^{1}$ Sem adição de cálcio. ${ }^{\S}$ Média de três repetições.

O lactato de cálcio, associado ou não ao agente seqüestrante citrato de sódio, foi o sal que apresentou menor perda durante o processamento do "leite" de soja. Entretanto, as amostras adicionadas deste sal apresentaram separação de fases. O produto adicionado de cálcio quelato perdeu cerca de $6 \%$ e não apresentou separação de fases e nem precipitação. Segundo os trabalhos de ASHMEAD [2], este mineral orgânico apresenta ótima biodisponibilidade (44\%) superando a do leite bovino que é de $27 \%$, além de ter alta solubilidade [12]. Assim, considerando os resultados encontrados, o cálcio quelato foi o mineral estudado mais indicado para o enriquecimento do extrato hidrossolúvel de soja.

\section{2 - Análise sensorial}

As médias dos atributos sensoriais avaliados neste estudo pela equipe treinada são apresentadas na Tabela 3. Observa-se nesta tabela que os atributos gredosidade e "beany flavour" não tiveram diferenças significativas entre as médias das amostras de "leite" de soja enriquecido com diferentes sais de cálcio. Em relação ao atributo sabor estranho, as amostras com cálcio quelato e lactato de cálcio tiveram as maiores médias; 4,37 e 4,24 respectivamente, numa escala não estruturada de nove pontos. As médias mais altas do atributo corpo foram atribuídas às amostras com lactato de cálcio com e sem citrato e foscalmix.

$\mathrm{Na}$ análise sensorial realizada com a equipe treinada o "leite" de soja com adição dos sais lactato de cálcio, com e sem citrato de sódio, e cálcio quelato foram os que tiveram pior qualidade global, com médias variando de 4,22 a 5,31, considerando a escala não estruturada de nove pontos (Tabela 3). Segundo HENDLER [13] o carbonato de cálcio é a forma mais concentrada e mais barata, porém HIROTSUKA et al. [14] mostraram que tanto o citrato, o gluconato quanto o carbonato de cálcio têm baixa solubilidade, não sendo indicados para a fortificação de alimentos.

TABELA 3. Médias dos atributos sensoriais para amostras de "leite de soja"

\begin{tabular}{|c|c|c|c|c|c|}
\hline Amostra/atributos ${ }^{\S}$ & Gredosidade & $\begin{array}{c}\text { Sabor } \\
\text { estranho }\end{array}$ & Corpo & $\begin{array}{l}\text { "Beany } \\
\text { flavour" }\end{array}$ & $\begin{array}{l}\text { Qualidade } \\
\text { global }\end{array}$ \\
\hline "Leite" de soja sem $\mathrm{Ca}^{++}$ & 2,0 & $1,6^{\circ}$ & $3,9^{\circ}$ & 1,8 & $6,6^{\mathrm{ab0}}$ \\
\hline $\begin{array}{l}\text { Adicionado de Carbonato de } \mathrm{Ca}^{+*} \\
\text { com citrato de sódio }\end{array}$ & 2,2 & $1,5^{\mathrm{c}}$ & $4,7^{\mathrm{bcd}}$ & 2,1 & $6,9^{\mathrm{ab}}$ \\
\hline $\begin{array}{l}\text { Adicionado de Fosfato de tricálcio } \\
\text { com citrato de sódio }\end{array}$ & 2,2 & $1,9^{\mathrm{ab}}$ & $4,9^{\circ}$ & 2,4 & $6,5^{a b c}$ \\
\hline $\begin{array}{l}\text { Adicionado de Lactato } \mathrm{Ca}^{++} \mathrm{com} \\
\text { Citrato de sódio }\end{array}$ & 2,5 & $2,9^{\mathrm{ab}}$ & $7,3^{\mathrm{a}}$ & 2,3 & $5,3^{\text {cd }}$ \\
\hline Adicionado de Cálcio Quelato & 2,7 & $4,4^{\mathrm{a}}$ & $5,0^{20}$ & 2,6 & $4,7^{\mathrm{cd}}$ \\
\hline Adicionado de Foscalmix @ & 2,4 & $1,3^{\mathrm{c}}$ & $6,3^{a b c}$ & 2,3 & $7,1^{\mathrm{a}}$ \\
\hline Adicionado de Carbonato de $\mathrm{Ca}^{+*}$ & 2,1 & $2,6^{\mathrm{ab}}$ & $4,5^{\mathrm{cd}}$ & 2,3 & $6,4^{\mathrm{abc}}$ \\
\hline Adicionado de Fosfato tricálcio & 2,1 & $1,5^{\mathrm{bc}}$ & $4,8^{\mathrm{ab}}$ & 1,9 & $7,4^{\mathrm{a}}$ \\
\hline Adicionado de Lactato de $\mathrm{Ca}^{++}$ & 3,1 & $4,2^{\mathrm{a}}$ & $6,2^{a b c}$ & 3,1 & $4,2^{d}$ \\
\hline
\end{tabular}
savaliados em escala não estruturada de nove pontos.

Com os resultados da avaliação da perda do cálcio foi possivel verificar que o cálcio quelato foi o sal mais adequado para o enriquecimento do "leite" de soja, dentre os avaliados, pois a perda deste mineral durante o processamento foi inferior a dos demais, exceto pelo lactato de cálcio. Entretanto, este último apresentou separação de fases. Os resultados da avaliação sensorial mostraram que o cálcio quelato não apresentou boa qualidade global, porém, devido à alta solubilidade e alta biodisponibilidade deste elemento, recomenda-se o seu uso para o enriquecimento do "leite". Recursos tecnológicos como por exemplo a adição de aromas naturais à bebida, podem ser empregados visando mascarar o sa- 
bor estranho relatado pela equipe de provadores treinados. Testes preliminares já realizados confirmaram que a adição de aromatizantes contribuiu positivamente para eliminar o referido sabor estranho.

A quantidade de cálcio da bebida de EHS considerando a IDR é apresentada na Tabela 4. Uma vez que o produto foi adicionado de cálcio para atingir 30\% da IDR e, com a perda do mineral durante o processamento, o teor final alcançou 28\% da IDR. A Legislação Brasileira exige, para produtos alimentícios na forma líquida, pelo menos $15 \%$ da IDR, ou seja, o produto final deve ter pelo menos $120 \mathrm{mg}$ de cálcio em cada $100 \mathrm{~mL}$. No caso do presente estudo a quantidade de cálcio no "leite" de soja disponibilizado para a subseqüente etapa de aromatização ultrapassou as recomendações da legislação e, desta forma, pode ser considerado um produto enriquecido.

TABELA 4. Teor de cálcio das amostras e porcentagem (\%) da Ingestão Diária Recomendada (IDR).

\begin{tabular}{ccc}
\hline \multicolumn{3}{c}{ "Leite" de soja } \\
\hline Cálcio & Sem adição de cálcio & Adicionado de cálcio quelato \\
$(\mathrm{mg} / 100 \mathrm{~g})$ & $8,6+2$ & $225,1+6$ \\
$\%$ IDR & 1,0 & 28,1 \\
\hline
\end{tabular}

A quantidade de cálcio no produto final é importante, mas a sua biodisponibilidade no organismo é fundamental para que o produto forneça quantidades adequadas do elemento ao consumidor [27]. É fundamental que o cálcio seja não somente ingerido, mas também absorvido em quantidades adequadas para a realização das funções metabólicas. Estudos futuros devem priorizar tal aspecto.

\section{4 - CONCLUSÕES}

A partir dos resultados obtidos nos experimentos realizados neste estudo, pode-se concluir que:

- o lactato de cálcio seguido do cálcio quelato foi o sal que apresentou menor perda durante o processamento do "leite" de soja enriquecido com cálcio, porém houve separação de fases nas amostras formuladas com o mesmo;

- a qualidade global do "leite" de soja adicionado de fosfato tricálcio alcançou a melhor avaliação pela equipe de provadores treinados;

- considerando as características sensoriais, o produto adicionado de cálcio quelato apresentou sabor estranho mais intenso, porém a adição de aromas naturais sabor chocolate, morango e baunilha pode mascarar tal atributo, sendo, portanto, o indicado para o enriquecimento.

\section{5 - REFERÊNCIAS BIBLIOGRÁFICAS}

[1] A.A.C.C. American Association of Cereal CHEMISTS. 1990. Approved Methods, $8^{\text {th }}$ Ed. v.II. St.Paul (método
46-12).

[2] ASHMEAD, H.D. 1996. Nutrição \& Aminoácidos quelatos. 1ed. Rio de Janeiro. Attar.

[3] A.O.C.S. Official Methods and recomended Practices of the American Oil Chemist's Society. 1994. 4.ed. Champaign.

[4] BRINK, E.J.; DEKLER, P.R.; VAN BERESTEIJN, E.C.; BEYNEM, A.C. Bioavailability of magnesium and calcium from cow's milk and soya-bean beverage in rats. British Journal Nutrition, n. 68, p. 271-282, 1992.

[5] CASÉ, F.; DELIZA, R.; ROSENTHAL, A.; CABRAL, L.C.; FREITAS, S.C. "Leite" de soja enriquecido com cálcio: avaliação da perda do mineral no processamento. In: $4^{\circ}$ SIMPÓSIO LATINO AMERICANO DE CIÊNCIA DE ALIMENTOS, 2001, Campinas. Livro de Resumos. Campinas - SP: R. Vieira Gráfica e Editora Ltda., 2001. p. 355-355.

[6] COZZOLINo, S.M.F. Biodisponobilidade de minerais. Nutrição PUCCAMP, v. 10, n. 2, p. 87-98, 1997.

[7] DELIZA, R.; SERNA SALDIVAR, S.; GERMANI, R.; BENASSI, V.T.; CABRAL, L.C. The effects of colored textured soybean protein (TSP) on sensory and physical attributes of ground beef patties. Journal of Sensory Studies, v. 17, n. 2, p. 121-132, 2002.

[8] KAJI, N.; MIZUSAWA, S.; SAHASHI, M.; TSUCHIDA, T. 1995. Calcium-enriched drink and method for producing the same. United States Patent n. 5609898.

[9] KATZ, F. Research priorities more toward healthy and safe. Food Technology, v. 54, n. 12, p .42-44, 2000.

[10] KRAUSE, M.V.; MAHAN, L.K. 1991. Alimentos, Nutrição e Dietoterapia. 7ed. Roca. 978 p.

[11] KROEZE, J.H.A. The perception of complex taste stimuli. In: MCBRIDE, R.L.; MACFIE, H.J.H. Psychological basis of sensory evaluation. Elsevier Applied Science, London. p. 41-68, 1990.

[12] HEANEY, R.P.; DOWELL, M.S.; RAFERTY, K.; BIERMAN, J. Bioavailability of the calcium in fortified soy imitation milk, with some observations on method. Am J Clin Nutr., v. 71, p. 1166-9, 2000.

[13] HENDLER, S.S. A Enciclopédia de Vitaminas e Minerais. Rio de Janeiro: Editora Campos, p. 114-120, 1994.

[14] HIROTSUKA, M.; TANIGUCHI, H.; NARITA, H. KITO, M. Calcium Fortification of Soy Milk with CalciumLecithin Liposome System. Journal of Food Science, n. 49, 1984

[15] HORWITZ, W. 2000. Official Methods of Analysis Of AOAC International. 17ed. Gaithersburg, Maryland.

[16] INSTITUTO ADOLFO LUTZ. Normas Analíticas do Instituto Adolfo Lutz. Vol. 1. Métodos Químicos e Físicos para Análise de Alimentos. São Paulo: IAL. $3^{\text {a }}$ ed., 371 p., 1986.

[17] NEVEN, L. Isoflavones - an overview of benefits for health and market. Agro-Food Industry HiTechnology, p. 39-41, 1998.

[18] MOSQUiN, M.C.A.; PEIXOTO, E.C.; MAGALHÃES, G.; MONTEIRO, R.R. Enriquecimento com Cálcio do leite submetido a Pasteurização. Boletim Informativo Purac, 1996.

[19] NELSON, A.I.; STEINBERG, M.P.; WEI, L.S. 1978. Whole soybean foods for home and village use. INTSOY n.14 may.

[20] RASYID, F.; HANSEN, P.M.T. Stabilization of soy milk fortified with calcium gluconate. Food Hydrocolloids, v. 4, n. 5, p. 415-422, 1991. 
[21] RIAZ, M.N. Soybeans as functional foods. Cereal Foods World, v. 44, n. 2, p. 88-92, 1999.

[22] SHEPHERD, R. Does taste determine consumption? Understanding the psychology of food choice. In: FREWER, L.J.; RISVIK, E.; SCHIFFERSTEIN, H. (Eds.). Food, People and Society - A European Perspective of Consumers' Food Choice. Springer, Berlin. p. 117-130, 2001.

[23] SlightowleR, H.; DAVIES, J. The Vegan Dairy. Nutrition and Food Science, v. 2, n. 2, p. 3153-157, 1998.

[24] TIJSSELiNG, L. Calcium Fortification of Soy Milk. Boletim informativo Purac, 1997.

[25] TOMOTADA, O.; SHOJI K.; MOTHIZEIKI, K. Influences of Calcium and $\mathrm{pH}$ on Protein Solubility in Soybean Milk. Biosci. Biocheistry, v. 57, n. 1, p. 24-28, 1993.
[26] YAZICI, F.; ALVAREZ, V.B.; HANSEN, P.M.T. Fermentation and Properties of Calcium-fortified Soy Milk Yogurt. Journal of Food Science, v. 62, n. 3, 1997.

[27] XU, X.; WANG, H.; MURPHY, P.A.; COOK, L.; HENDRICH, S. Daidzein is a more bioavailable soymilk isoflavone than is genistein in adult women. Journal of Nutrition, 124: 825-832, 1994.

[28] WEAVER, C.M; PROULX, W.R.; HEANEY, R. Choices for achieving adequate dietary calcium with a vegetarian diet. American Journal of Clinical Nutrition, n. 70 (suppl): 543S-8S, 1999.

[29] WEINGARTNER, K.E.; NELSON, A.I.; ERDMAN, JR. J.W. Effects of Calcium Addition on Stability and Sensory Properties of soy Beverage. Journal of Food Science, n. 48, 1983. 